\title{
Robotic Partial Excision of Levator-Ani Muscle for Locally Advanced Low Rectal Cancer Invading Ipsilateral Pelvic Floor
}

\author{
Seung Yoon Yang, Nam Kyu Kim \\ Department of Surgery, Severance Hospital, Yonsei University College of Medicine, Seoul, Korea
}

Tumors at the level of the anorectal junction had required abdominoperineal resection (APR) to achieve an adequate resection margin. However, in the cases of tumor invading ipsilateral levator-ani muscle (LAM), en-bloc resection of the rectum with LAM including tumor would be possible. This video is to show the critical anatomic steps of this procedure. A video was produced from the robotic right partial excision of LAM (PELM) performed in a 57-year-old female patient with rectal cancer at $3 \mathrm{~cm}$ from the anal verge, invading the ipsilateral anorectal ring, who had received neoadjuvant chemoradiotherapy. The patient discharged at postoperative day 8 without complication. The pathology of the surgical specimen revealed ypT3N1bM0. The secure resection margin from the tumor was achieved. Robotic PELM is the sphincter-preserving technique that can be an alternative treatment option for low rectal cancer invading the ipsilateral LAM, which has been an indication for APR or extralevator APR.

Keywords: Rectal neoplasms; Robotic surgery; Levator-ani muscle

Abdominoperineal resection (APR) is recommended for tumors extending beyond the intersphincteric plane in patients with low rectal cancer, even though recent advances in surgical techniques and multidisciplinary treatments have led to the possibility of sphincter preservation [1]. However, in the cases of tumor invading ipsilateral levator-ani muscle (LAM) and intact external sphincter, en-bloc resection of the rectum with LAM, including tumor, would be possible [2]. Based on this idea, preliminary cadaveric study, the author started this procedure since 2011 under the consent of the patient with the inclusion/exclusion criteria shown below.

Received: April 15, 2020 • Accepted: June 29, 2020

Correspondence to: Nam Kyu Kim, M.D.

Division of Colon and Rectal Surgery, Department of Surgery, Severance

Hospital, Yonsei University College of Medicine, 50-1 Yonsei-ro,

Seodaemun-gu, Seoul 03722, Korea

Tel: +82-2-2228-2100, Fax: +82-2-313-8289

E-mail:namkyuk@yuhs.ac

ORCID: https://orcid.org/0000-0003-0639-5632

(C) 2020 The Korean Society of Coloproctology

This is an open-access article distributed under the terms of the Creative Commons Attribution NonCommercial License (https://creativecommons.org/licenses/by-nc/4.0) which permits unrestricted non-

commercial use, distribution, and reproduction in any medium, provided the original work is properly cited.

\section{Inclusion criteria}

- Qualitative diagnosis: a pathological diagnosis of adenocarcinoma of the rectum

- Tumor invading the ipsilateral LAM at the level of the anorectal ring on pretreatment magnetic resonance imaging (MRI)

- Tumor occupying less than half of the bowel lumen on preoperative colonoscopy

- Suitability for curative surgery and age of $>19$ and $<75$ years

\section{Exclusion criteria}

- Preoperative imaging examination results showing distant metastasis

- Tumor invading beyond midline of the ipsilateral LAM at the level of the anorectal ring on pretreatment MRI

- Tumor occupying more than half of the bowel lumen on preoperative colonoscopy

- Patients with poor anal function

The partial excision of LAM (PELM) technique enables preserving the anal sphincter function with obtaining oncologic clearance and avoiding permanent colostomy in those patients. The purpose of this video is to show the critical anatomic steps of this procedure. The Institutional Review Board (IRB) of Severance 
Hospital approved this study and informed consent was waived by the IRB (No. 4-2015-0815).

A video was produced from the robotic intersphincteric resection with the right PELM procedure performed in a 57-year-old female patient with low rectal cancer at $3 \mathrm{~cm}$ from the anal verge, invading the anorectal ring, who had received neoadjuvant chemoradiotherapy (nCRT). After restaging the patient with an MRI, the surgery was scheduled at 8 weeks after nCRT completion. The patients underwent standard bowel preparation using 4 L of Colyte (Taejoon Pharm., Seoul, Korea) the day before the surgery and were administered antibiotic prophylaxis before the incision. The surgery was carried out under general anesthesia with the patient in the lithotomy position. The patient was placed in the Lloyd-Davies of Pencoed position with a steep Trendelenburg position. The robotic PELM consisted of 2 phases; abdominal and perineal phase. The abdominal phase started with medial to the lateral mobilization of the left colon, including full mobilization of the splenic flexure, central vessel ligation, and total mesorectal excision (TME), followed by dissection toward the LAM. Break down further, the abdominal phase consisted of 2 phases. The first phase was the colonic phase, which comprised ligation of inferior mesenteric vessels and mobilization of the left colon and splenic flexure. The second phase was the pelvic phase, which constituted pelvic dissection using the TME principles. Our technique for robotic TME using the da Vinci Surgical System (Intuitive Surgical, Sunnyvale, CA, USA) has been described previously $[3,4]$. Dissection continued to the pelvic floor consisting of the LAM. The puborectalis muscle was exposed at the contralateral side of the tumor. Then, dissection and excision of the LAM on the ipsilateral side of the tumor were performed with an adequate margin. It is essential to stop the dissection just above the origin of the LAM, and not to dissect the mesorectum off the LAM. Therefore, dissection was performed down to the coccyx, where the origin of the LAM was identified. The LAM was first transected from the coccyx slightly distally to its tip, and then laterally with adequate circumferential resection margin; this was done under direct vision using monopolar curved scissors to enter the ischiorectal fat. After the completion of pelvic dissection, the perineal phase was started. The patient's hips were flexed to allow better access to the perineum. An incision was made at the intersphincteric groove on the side of the tumor, to approach the intersphincteric space. The dissection then continued among the internal and external anal sphincter to the level approximately 0.5 to $1 \mathrm{~cm}$ below the tumor according to distance from the anal verge, where the dissection turned transversally to include the deep part of the external sphincter until the ischiorectal fossa fat could be visualized. The dissection continued in a cephalic direction to include the involved part of the LAM, from where the pelvic cavity is entered. On the contralateral side of the tumor, the incision was made just above the dentate line, and the dissection was in a plane medial to the internal sphincter, which allowed entering the pelvic cavity medially to the puborectalis muscle, in which the distal rectum was divided in a sleeve fashion. After the total excision of the rectum, the rectum and sigmoid colon were extracted through the anus and transected. A sleeve-fashioned resection was performed to the distal rectum; after that, bowel continuity was restored using a hand-sewn coloanal anastomosis (CAA). A diverting loop ileostomy was created.

The patient resumed a soft diet at postoperative day (POD) 4 without any problems and discharged at POD 8 without any complication. The pathology of the surgical specimen revealed perirectal fat tissue invasion with 2 regional lymph node metastases (ypT3N1bM0). Neither lymphovascular invasion nor perineural invasion was found. The secure resection margin from the tumor was achieved, especially the circumferential resection margin, which was the primary intention of PELM.

PELM is a new surgical treatment alternative to APR or extralevator APR for low rectal cancer invading the ipsilateral LAM at the level of the anorectal ring. Furthermore, the benefits of this sphincter preserving surgery can be maximized when combined with robotic surgery, which enables more precise surgery. However, the long-term outcomes of this procedure still need to be assessed to confirm its oncologic and functional safety.

\section{CONFLICT OF INTEREST}

No potential conflict of interest relevant to this article was reported.

\section{SUPPLEMENTARY MATERIALS}

Supplementary video clip can be found via https://doi.org/10.3393/ ac.2020.06.29.v001.

\section{REFERENCES}

1. Smith JJ, Garcia-Aguilar J. Advances and challenges in treatment of locally advanced rectal cancer. J Clin Oncol 2015;33:1797-808.

2. Noh GT, Han J, Cheong C, Han YD, Kim NK. Novel anal sphincter saving procedure with partial excision of levator-ani muscle in rectal cancer invading ipsilateral pelvic floor. Ann Surg Treat Res 2017;93:195-202.

3. Bae SU, Min BS, Kim NK. Robotic low ligation of the inferior mesenteric artery for rectal cancer using the firefly technique. Yonsei Med J 2015;56:1028-35.

4. Park YA, Kim JM, Kim SA, Min BS, Kim NK, Sohn SK, et al. Totally robotic surgery for rectal cancer: from splenic flexure to pelvic floor in one setup. Surg Endosc 2010;24:715-20. 